\title{
Clinical value of an electromagnetic navigation system for CT-guided percutaneous lung biopsy of peripheral lung lesions
}

\author{
Yao Zhang ${ }^{1 \#}$, Haozhe Huang ${ }^{2 \#}$, Shuhui Cao ${ }^{1}$, Jiajun Teng ${ }^{1}$, Yue Wang ${ }^{1}$, Xuxinyi Ling ${ }^{1}$, Yan Zhou ${ }^{1}$, \\ Jianlin $\mathrm{Xu}^{1}$, Wentao $\mathrm{Li}^{2}$, Hua Zhong ${ }^{1}$ \\ ${ }^{1}$ Department of Pulmonary, Shanghai Chest Hospital, Shanghai Jiao Tong University, Shanghai, China; ${ }^{2}$ Department of Interventional Radiology, \\ Fudan University Shanghai Cancer Center, Xuhui, Shanghai, China \\ Contributions: (I) Conception and design: Y Zhang, H Huang, S Cao, H Zhong; (II) Administrative support: W Li, H Zhong; (III) Provision of study \\ materials or patients: W Li, H Zhong, J Teng; (IV) Collection and assembly of data: Y Zhang, Y Wang, X Ling; (V) Data analysis and interpretation: \\ Y Zhang, W Li, J Xu, Y Zhou; (VI) Manuscript writing: All authors; (VII) Final approval of manuscript: All authors. \\ \#These authors contributed equally to this work. \\ Correspondence to: Hua Zhong, MD. Department of Pulmonary, Shanghai Chest Hospital, Shanghai Jiao Tong University, Huaihai West Road No. \\ 241, Shanghai 200030, China. Email: eddiedong8@hotmail.com; Wentao Li, MD. Department of Interventional Radiology, Fudan University \\ Shanghai Cancer Center, 270 Dongan Road, Xuhui, Shanghai 200032, China. Email: wentaoli@fudan.edu.cn.
}

\begin{abstract}
Background: The purpose of this study was to retrospectively evaluate the clinical value of an electromagnetic navigation system for CT-guided percutaneous lung biopsy of peripheral lung lesions.

Methods: This was a retrospective study. Patients with peripheral lung lesions in our institution between January 2019 and December 2020, who underwent lung biopsy assisted by the electromagnetic navigation system were included in Group A, and those who underwent lung biopsy using conventional CT-guided percutaneous lung biopsy were included in Group B. The general features and clinical and technical information of each patient were collected and evaluated in both groups.

Results: A total of 141 patients were included in Group A (78 males and 63 females; median age, 65 years; range, 32-79 years), and 96 patients were included in group B (57 males and 39 females; median age, 65 years; range, 34-80 years). The technical success rate was $100 \%$ in both groups. The technical efficacy rate was 92.9\% and $90.6 \%$ in Groups A and B $(\mathrm{P}=0.525)$, respectively. There was no significant difference in surgical time and the number of CT scans between the two groups, and only grade 1-2 complications occurred in the patients.
\end{abstract}

Conclusions: The electromagnetic navigation system is an effective and safe auxiliary tool for CT-guided percutaneous lung biopsy of peripheral lung lesions.

Keywords: Lung lesions; CT-guided biopsy; electromagnetic navigation; lung biopsy; safety; efficiency

Submitted Mar 09, 2021. Accepted for publication Jul 11, 2021.

doi: $10.21037 /$ jtd-21-395

View this article at: https://dx.doi.org/10.21037/jtd-21-395

\section{Introduction}

Lung cancer is the leading cause of cancer-related deaths in both males and females worldwide (1). Most patients have entered the advanced stage at diagnosis and have lost the opportunity for a complete cure. Thus, the early detection of lung cancer is particularly important (2). Computed tomography (CT)-guided percutaneous lung biopsy are widely used in clinical practice for obtaining tissue diagnoses from peripheral lung nodules. The technology has a high diagnostic accuracy $(3,4)$. However, the surgery cannot be monitored with real-time imaging, and thus relies on the surgeon's experience. Many complications such as pneumothorax and hemoptysis may arise after biopsy (5). Repeating CT scans can also cause significant radiation-related damage to patients.

Previous studies have reported that electromagnetic 

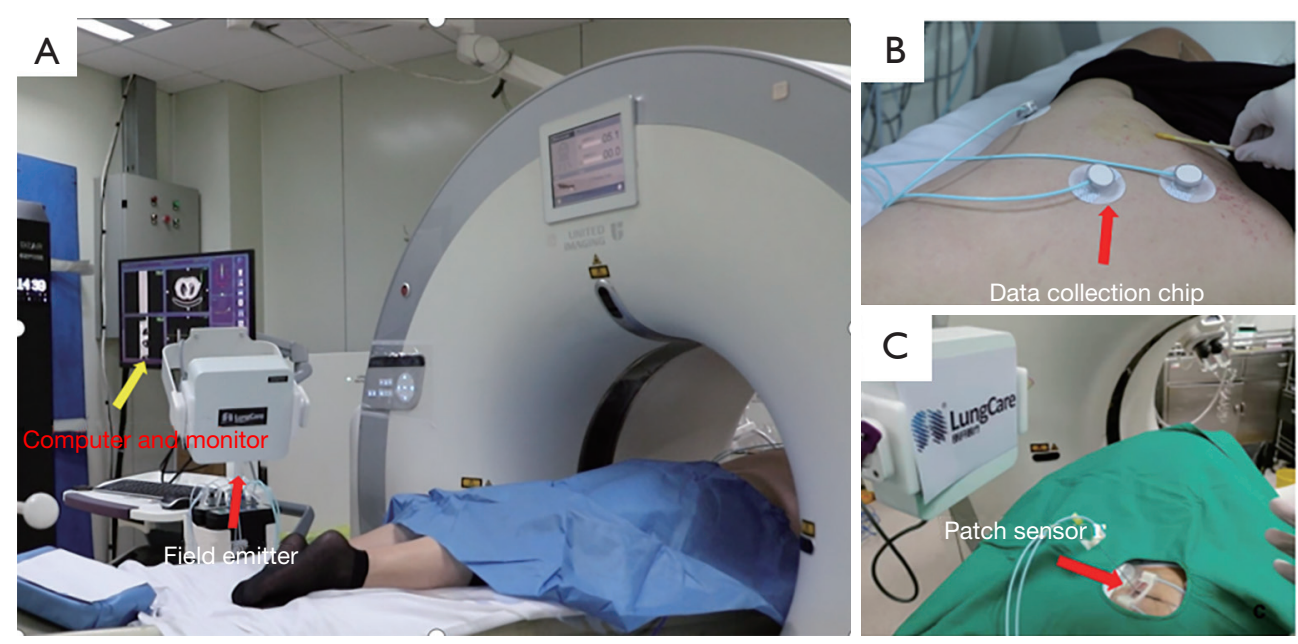

Figure 1 The components of Electromagnetic navigation system components. (A) Computer and monitor (yellow arrow), field emitter (red arrow); (B) data collection chip (arrow); (C) patch sensor (arrow).

navigation system-assisted bronchoscopy can be used safely and efficiently to diagnose lung lesions (6). The technology uses electromagnetic tracking technology to obtain spatial navigation information in real time, thus making it possible to accurately use therapeutic and real-time navigation during surgery. Compared to conventional approaches, it provides many benefits such as increased accuracy, reduction of surgical times, and reduction of complications and risks for patients (7-9). Considering the effective use of the electromagnetic navigation system in bronchoscopy, we reasoned that the electromagnetic navigation system could also be used for CT-guided percutaneous lung biopsy.

In this study, we used the electromagnetic navigation system for CT-guided percutaneous lung biopsy of peripheral lung lesions, and the clinical value of this new technology was compared with conventional CT-guided percutaneous lung biopsy. We present the following article in accordance with the STROBE reporting checklist (available at https://dx.doi.org/10.21037/jtd-21-395).

\section{Methods}

\section{Study population}

Patients with peripheral lung lesions who underwent lung biopsy assisted by the electromagnetic navigation system (Group A) or used conventional CT-guided percutaneous lung biopsy (Group B) between January 2019 and December 2020 in our institution were included in the study. The inclusion criteria involved patients whose spiral CT showed peripheral lung lesions, which were suspected of lung cancer. All patients were over 18 years of age, and written informed consent for the CT-guided biopsy was obtained from each patient.

\section{Study procedure}

The study was conducted in accordance with the Declaration of Helsinki (as revised in 2013). The study was approved by institutional review board of Shanghai Chest Hospital (KS1814), and written informed consent was taken from all patients before invasive procedures.

All biopsy procedures were conducted under local anesthesia by the same physician, who had more than 20 years of experience. All patients underwent coaxial fine needle aspiration or core biopsy according to the physician's decision. The physician also selected the suitable size of the biopsy needle, which ranged from 18-20 gauge, according to the condition of the lesions. All procedures were performed under CT guidance. The following parameters were used: image thickness $=1.5 \mathrm{~mm}, \mathrm{~mA}=234$, and $\mathrm{KvP}$ $=120$. The CT scan was performed by two radiologists with more than 15 years of experience in radiology. All the CT images were obtained at end expiration.

In group A, patients with peripheral lung lesions were treated with an electromagnetic navigation system (Lung Care; China, Figure 1) for CT-guided percutaneous lung biopsy. Before surgery, the patients were subjected to a CT scan. The resulting images were uploaded to an electromagnetic navigation system and then after reviewing the images, a proper puncture route was selected and 

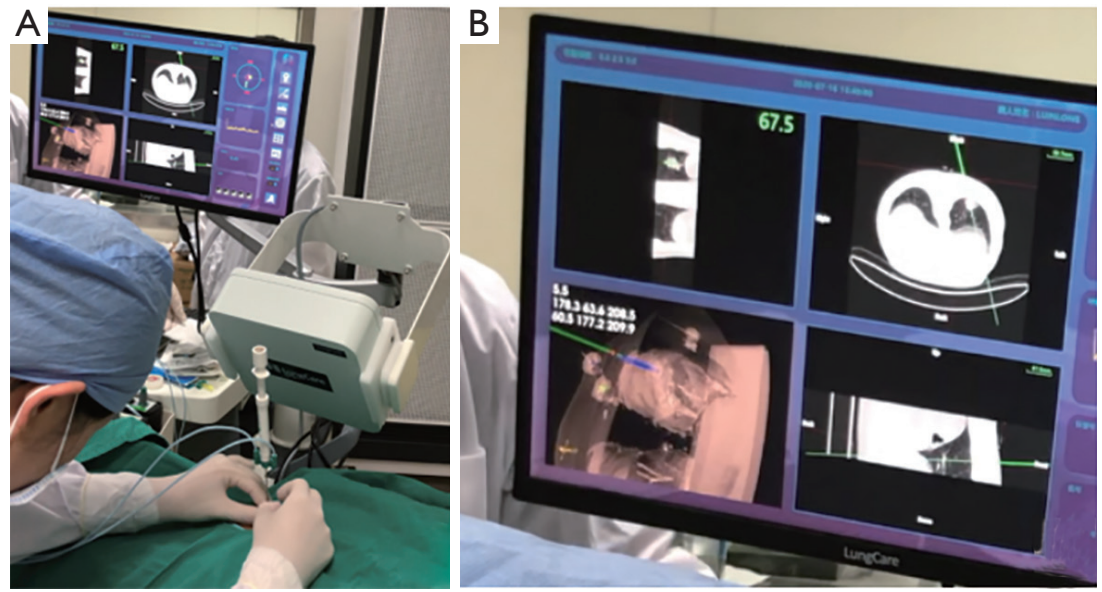

Figure 2 The electromagnetic navigation system guided lung biopsy. (A) The operator can visualize the needle advancement using the electromagnetic navigation system in real time; (B) the enlarged view of the screen.
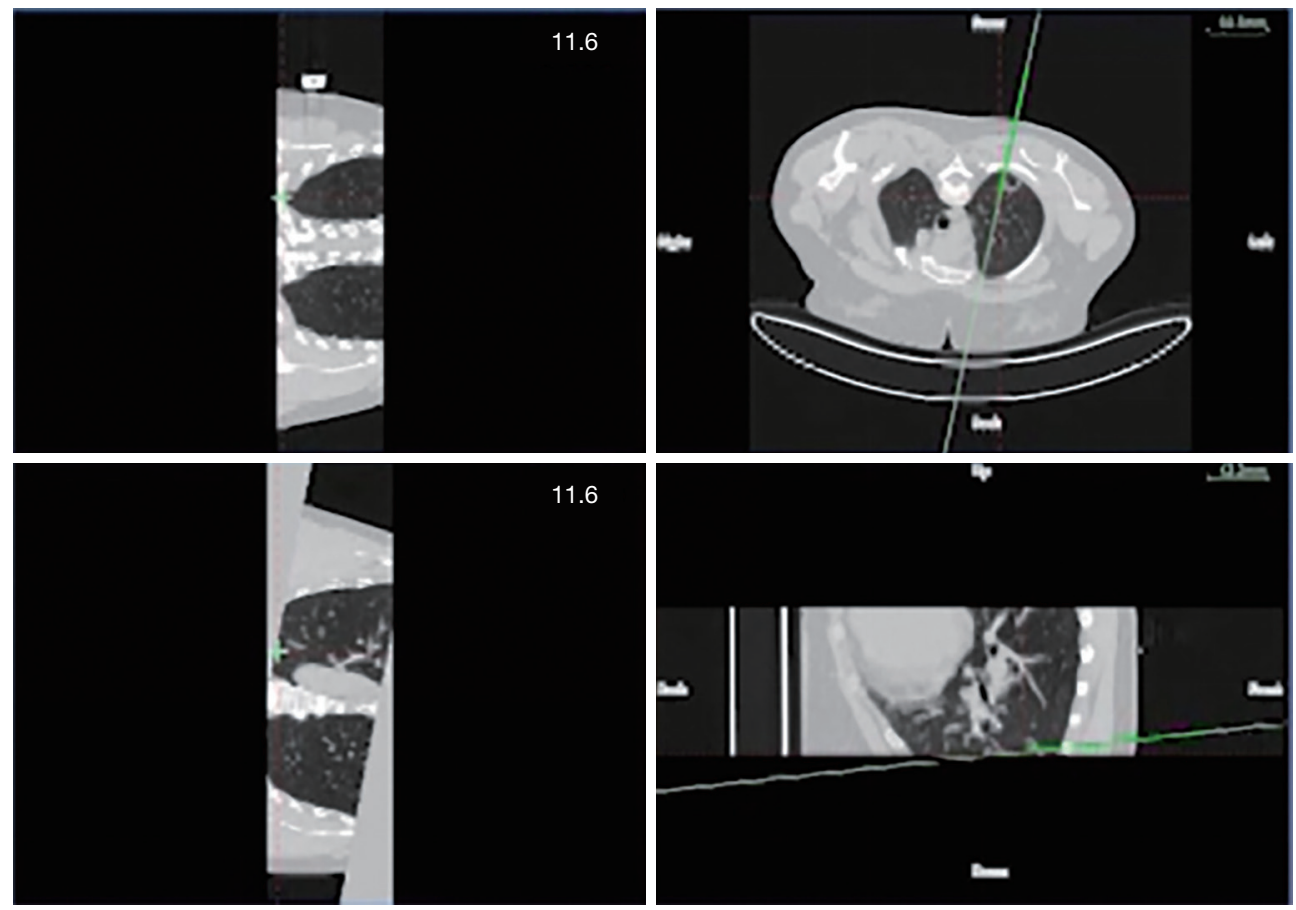

Figure 3 The electromagnetic navigation system's screen capture, which shows the position of the digital tip (cross) of the biopsy needle and needle route (line) in the axial and sagittal planes.

marked on the skin. The patients were told to assume a position according to the original CT scan, then the data collection chip was added and the patch sensor was installed near lesions on the skin to connect the electromagnetic navigation system, and the biopsy needle was then connected to the sensor. The electromagnetic navigation system superimposes the needle tip on the pre-procedural CT dataset, and then guided the biopsy needle to the target tissue according to the planned puncture route in real time (Figure 2). The system generated images viewed as axial, sagittal, coronal, or oblique to assist placement of the puncture needle (Figure 3). When the system showed that 
the needle tip was within the target tissue, CT scanning was again used to confirm the needle placement to obtain tissues for cytopathological or histopathological analyses.

In group B, patients with peripheral lung lesions were operated using conventional CT-guided biopsy. Before the procedure began, the surgeon used CT images in planning the lesion position and biopsy route. The surgeon then slowly advanced the needle. After using the CT scan to confirm that the needle tip was within the lesion, the tissues were obtained using a suitable biopsy needle.

The number of obtained specimen depended on lesion characteristics and surgeon's experience. All biopsy tissues were submitted for pathological analysis. If such examination failed to yield the diagnosis, we would take a second biopsy or perform the 3-6 months follow-up according to the patient condition.

\section{Data collection}

All patients had a CT scan, which was conducted after the procedure to check for complications. We recorded clinical and technical information in both groups. In addition, we evaluated the precise performance of the electromagnetic navigation system-assisted CT-guided lung biopsy and conventional CT-guided percutaneous lung biopsy in disease diagnosis, and compared the precise of disease diagnoses in the two groups. Technical success was defined as completion of the biopsy procedure and acquisition of lesion tissues (i.e., the procedure was completed successfully and was not terminated).

Technical efficacy was defined as the acquisition of adequate pulmonary lesion tissues, which had a definite pathological diagnosis (whether the pathological results were benign or malignant). The technical inefficacy was defined as follows: (I) the tissue was obtained in amounts too small to make pathological slides; (II) the pathological results were benign, but further bronchoscopy biopsy/ surgery yielded malignant results; and (III) there were only uncertain cytopathological results.

Safety was evaluated according to the occurrence of related complications such as pneumothorax, hemoptysis, and bleeding. The complications were classified according to the Society of Interventional Radiology (SIR) Guidelines (10).

\section{Statistical analysis}

Continuous parameters are presented as the mean \pm standard deviation (SD) or median (range), compared using an independent $t$-test or Mann-Whitney $\mathrm{U}$ test between the two groups. The categorical variables are presented as the number and percentage, and the chi-square test was used to compare categorical variables. We used SPSS (SPSS statistical software for Windows, version 23; IBM, Chicago, IL, USA) to perform statistical analyses, and $\mathrm{P}<0.05$ was considered statistically significant.

\section{Results}

\section{Patient and lesion characteristics}

A total of 141 patients were included in Group A (78 males and 63 females; median age, 65 years; range, 32-79 years), and 96 patients were included in group B (57 males and 39 females; median age, 65 years; range, $34-80$ years). There was no statistical difference in the sex and age distribution of the two groups $(\mathrm{P}>0.05)$. The mean lesion maximum sizes were $19.8 \pm 6.4 \mathrm{~mm}$ for group $\mathrm{A}$ and $20.9 \pm 5.9 \mathrm{~mm}$ for group $B(P=0.211)$. There was no significant difference in the puncture position and lesion maximum diameter, lesion lobar location, distance from the pleura, and other lesion parameters, and there was no significant difference in the solidity of lesions between the two groups. In Group A, of the 141 patients, 86 were diagnosed as malignant, compared with 63 of 96 patients in group B, and there was no significant difference between the two groups. As many lesions only submitted for cytopathological analysis, there were no specific pathological subtypes. The specific characteristics of the patients and pulmonary lesions in both groups are summarized in Table 1.

\section{Technique and efficacy}

All patients successfully completed the procedure. The technical success rate was $100 \%$ in Groups A and B. There was no significant statistical difference in the surgical time and number of adjusted times in CT scanning between Groups A and B (Table 2). We found that the shortest surgical time was only three minutes in Group A. The electromagnetic navigation system was therefore a highly efficient tool for CT-guided percutaneous lung biopsy of peripheral lung lesions. There was no significant statistical difference in the distance between the skin entry point and the target lesion. The overall technical efficacy rate of biopsy were $92.9 \%$ for group A and $90.6 \%$ for group B $(\mathrm{P}=0.525)$. 
Table 1 Clinical characteristics of patients and their lesions

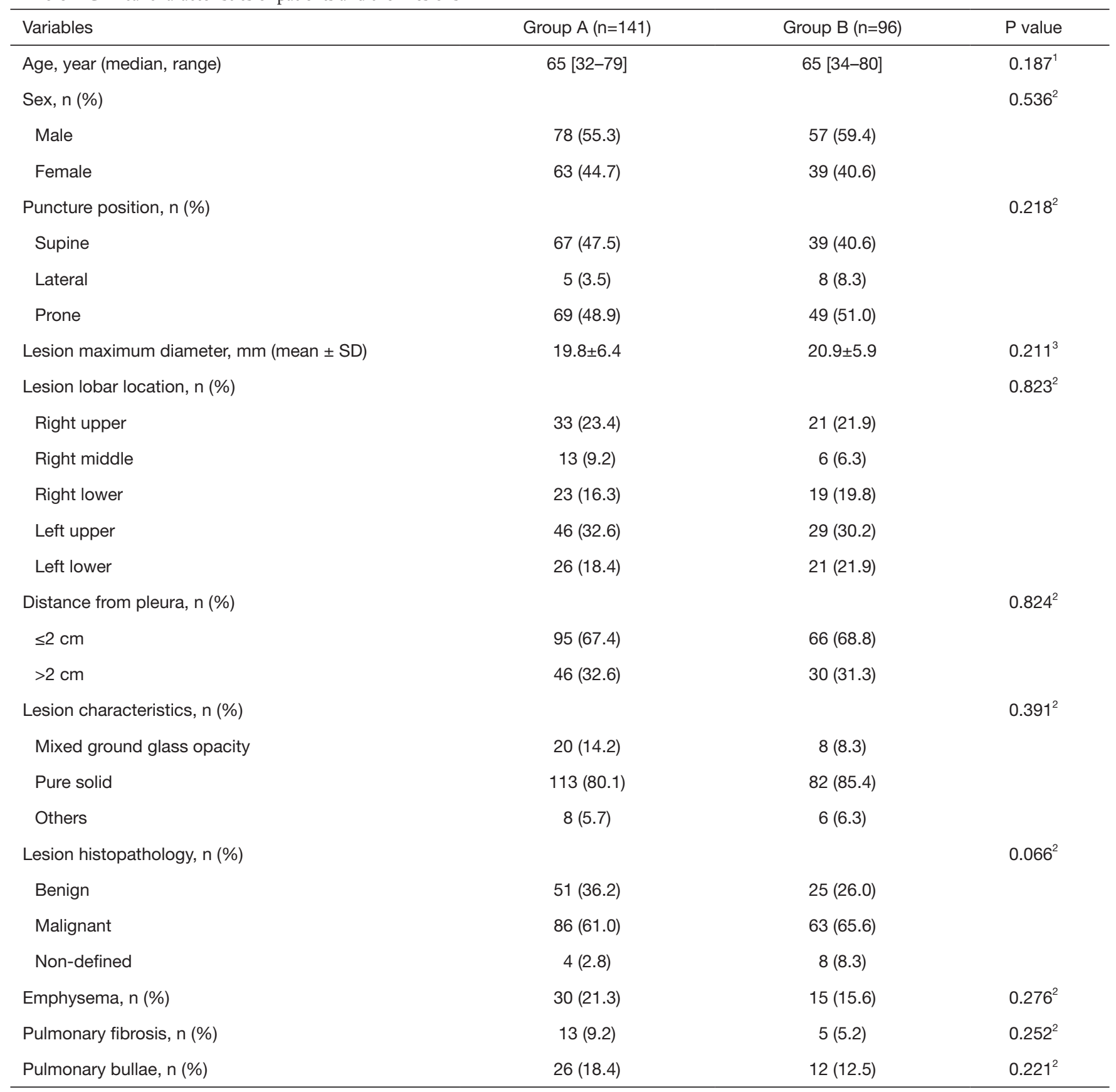

${ }^{1}$, Mann-Whitney $\mathrm{U}$ test; ${ }^{2}$, Chi-square test/Fishers' exact test; ${ }^{3}$, independent $t$-test.

\section{Complications}

Many kinds of observed complications are listed in Table 3, including pneumothorax [A vs. B: 29 (20.6\%) vs. 16 (16.7\%), $\mathrm{P}=0.452$ ], hemorrhage [A vs. B: 20 (14.2\%) vs. 17
(17.7\%), $\mathrm{P}=0.463$ ] and hemoptysis [A vs. B: 3 (2.1\%) vs. 2 (2.1\%), $\mathrm{P}=1.000]$. There was no significant difference in the incidences of all types of complications between the two groups, and no other serious complications occurred in both groups. We observed that there were only grade 1-2 
Table 2 Technical information

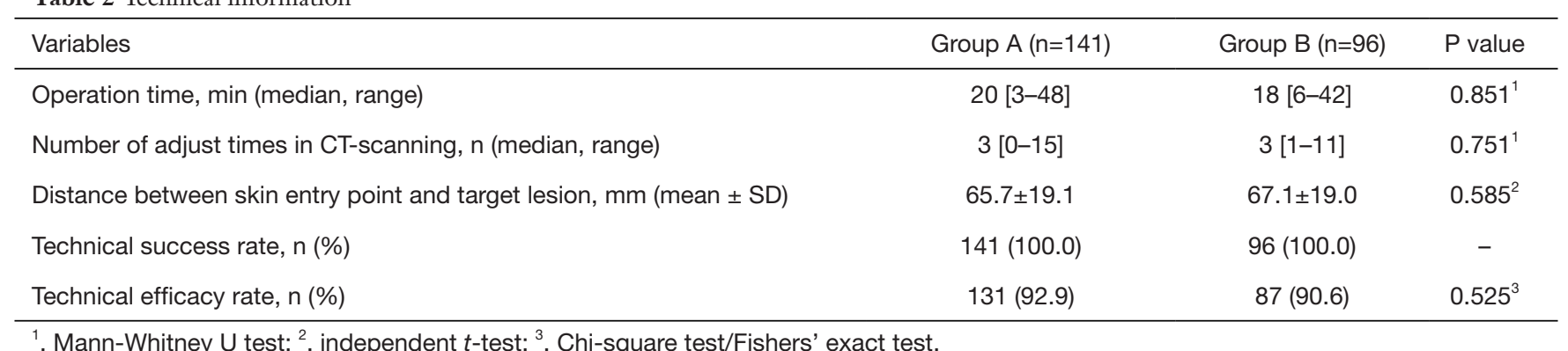

Table 3 Biopsy-related complications and grading by the Society of Interventional Radiology guidelines

\begin{tabular}{|c|c|c|c|}
\hline Complications & Group A (n=141) (\%) & Group B (n=96) (\%) & $P$ value* \\
\hline \multicolumn{4}{|l|}{ Grade of complications } \\
\hline Grade 1-2 & $52(36.9)$ & $35(36.5)$ & - \\
\hline \multicolumn{4}{|l|}{ Type of complications } \\
\hline Hemorrhage & $20(14.2)$ & $17(17.7)$ & 0.463 \\
\hline Hemoptysis & $3(2.1)$ & $2(2.1)$ & 1.000 \\
\hline
\end{tabular}

*, the $P$ value was calculated using the chi-square test.

minor complications in the patients.

\section{Discussion}

With the popular application of spiral CT in patients, a multitude of lung lesions have been found, with definite diagnosis in the early stage of these lung lesions being especially important. CT-guided percutaneous biopsies have been widely used in the diagnoses of peripheral lung lesions (11). Traditional biopsy methods only use repeated CT scans to determine the angle and depth for puncture surgery. During surgery, the technical success depends on the operator's personal experiences, so repeated CT scanning may be necessary to verify the correct needle position. The repeat CT scan also requires a long time and is prone to increase the occurrences of complications and radiation damage $(12,13)$. The electromagnetic navigation system is a new technique, which has been previously used to assist bronchoscopy for the diagnosis of lung cancer (14). However, it is necessary to improve the technical efficacy without the disadvantages of radiation and time consumption, to identify targeted positions in real time (15).

It is possible for the electromagnetic navigation system to locate the needle tip position in real time, and to overlay real-time instrument tracking information using CT-imaging (16). Based on this feature, the technical success and the probability of obtaining effective tissues are increased. Yarmus et al. (17) showed that navigation bronchoscopy combined with the electromagnetic guidance system increased the diagnostic yield, which uses electromagnetic tracking technology to obtain spatial navigation information in real time, thus making it possible to accurately use therapeutic and real-time navigation during surgery, but the technical is suitable for bronchus central lesions. In contrast to previous surgical procedures, which were only based on the operators' experiences, because of the real-time check on the needle tip position, the success of beginning surgeons has increased. Santos et al. (18) reported that electromagnetic navigation was successful in all patients, which was consistent with our results. The technical success rate was $100 \%$ and technical efficacy rate was $92.9 \%$ in our study, which showed that the electromagnetic navigation system was an efficient and safe auxiliary tool for CT-guided percutaneous lung biopsies of peripheral lung lesions. In a previous study by Grand et al. (19), 8 patients (30.8\%) failed to complete the biopsy 
procedure using electromagnetic navigation, the reasons for technical failures included technical difficulties and the surgeons' lack of experience. All of our surgeries were performed by experienced physicians and radiologists.

Grand et al. (19) compared the electromagnetic navigation system $(\mathrm{n}=26)$ to conventional CT fluoroscopy biopsies ( $n=34)$, and reported that there was no significant difference in procedural times between the two groups. The results were probably due to the inclusion criteria restricted to small masses $(<2.5 \mathrm{~cm})$ in the study, which is consistent with our results. There was also no significant difference in surgical time and in the number of CT scans in our study. The reason may be that electromagnetic navigation systemassisted CT-guided percutaneous lung biopsy involves a novel technology, so the operator needs to be very careful during surgery. In contrast, Grasso et al. (15) performed electromagnetic navigation-assisted CT-guided percutaneous lung biopsy on 197 patients, and found this procedure was superior to conventional CT-guided biopsies in terms of surgical times and numbers of CT scans, which was even better than our results. The reason may be that the numbers of patients enrolled in this study were higher than our study, so the surgeon became more proficient with continuous practice. Furthermore, the instrument we used was the first electromagnetic navigation system used in China.

Although the CT-guided percutaneous lung biopsy is an efficient and safe method to diagnose peripheral lung lesions, postoperative complications are inevitable. In terms of safety, there was no statistical difference in the occurrence of complications between the two groups $(\mathrm{P}=0.947)$. The most common complication in the two groups was pneumothorax $(29,20.6 \%)$ and hemorrhage (17, 17.7\%). According to a previous report, pneumothorax and pulmonary hemorrhage were the most common complications for CT-guided biopsy (20), which is consistent with our study results. In a study by Hiraki et al. (21), the overall pneumothorax percentage was $42.3 \%$, which was significantly higher than our results. Additionally, all complications occurring in both groups were divided into grades I-II using SIR Guidelines (10). Most complications were self-limiting and did not require further interventions.

In addition, the patient movement between preprocedural CT scan and needle tip placement may lead to inaccurate registration. In Grand's study (19), they use a multi-center CT scanner during acquisition of initial dataset to avoid the above problem. In our study, once the preprocedural CT scan accomplished and a proper puncture route was selected, we kept the patient from moving and let the patient breathe slowly and softly, and we used different angles CT image to show the position of the digital tip of the biopsy needle and needle route.

Although the electromagnetic navigation system is not superior to conventional CT guidance, the new technical is suitable for young doctor who was lack of experience in biopsy. And as experiences increase, we can improve the utilization efficiency of electromagnetic navigation system.

Inevitably, our study had several limitations. First, this study was a single center retrospective study, which may have had certain selection bias. Second, the number of patients was too small to show statistically significant differences between the two groups, so more patients are needed to confirm our results. Third, this navigation system (Lung Care) was a totally new system, so additional surgeries need to be performed in the future. Finally, we did not completely follow up patients with benign pathological diagnoses.

In conclusion, the clinical application of electromagnetic navigation was an effective and safe auxiliary tool for CT-guided percutaneous lung biopsy. We are presently conducting more extensive research to confirm our promising results.

\section{Acknowledgments}

Funding: This work was supported by the National Key Research and Development Program of China (Project No. 2017YFC0112700 and 2017YFC0112703) and the Joint Research Project of Emerging Frontier Technology in Municipal Hospital (Project No. SHDC12019127).

\section{Footnote}

Reporting Checklist: The authors have completed the STROBE reporting checklist. Available at https://dx.doi. org/10.21037/jtd-21-395

Data Sharing Statement: Available at https://dx.doi. org/10.21037/jtd-21-395

Peer Review File: Available at https://dx.doi.org/10.21037/ jtd-21-395

Conflicts of Interest: All authors have completed the ICMJE uniform disclosure form (available at https://dx.doi. org/10.21037/jtd-21-395). The authors have no conflicts of interest to declare. 
Ethical Statement: The authors are accountable for all aspects of the work in ensuring that questions related to the accuracy or integrity of any part of the work are appropriately investigated and resolved. The study was conducted in accordance with the Declaration of Helsinki (as revised in 2013). The study was approved by institutional review board of Shanghai Chest Hospital (KS1814), and written informed consent was taken from all patients before invasive procedures.

Open Access Statement: This is an Open Access article distributed in accordance with the Creative Commons Attribution-NonCommercial-NoDerivs 4.0 International License (CC BY-NC-ND 4.0), which permits the noncommercial replication and distribution of the article with the strict proviso that no changes or edits are made and the original work is properly cited (including links to both the formal publication through the relevant DOI and the license). See: https://creativecommons.org/licenses/by-nc-nd/4.0/.

\section{References}

1. Bray F, Ferlay J, Soerjomataram I, et al. Global cancer statistics 2018: GLOBOCAN estimates of incidence and mortality worldwide for 36 cancers in 185 countries. CA Cancer J Clin 2018;68:394-424.

2. Hirsch FR, Scagliotti GV, Mulshine JL, et al. Lung cancer: current therapies and new targeted treatments. Lancet 2017;389:299-311.

3. Drumm O, Joyce EA, de Blacam C, et al. CT-guided Lung Biopsy: Effect of Biopsy-side Down Position on Pneumothorax and Chest Tube Placement. Radiology 2019;292:190-6.

4. Heerink WJ, de Bock GH, de Jonge GJ, et al. Complication rates of CT-guided transthoracic lung biopsy: meta-analysis. Eur Radiol 2017;27:138-48.

5. Wu CC, Maher MM, Shepard JA. Complications of CT-guided percutaneous needle biopsy of the chest: prevention and management. AJR Am J Roentgenol 2011;196:W678-82.

6. Folch EE, Bowling MR, Gildea TR, et al. Design of a prospective, multicenter, global, cohort study of electromagnetic navigation bronchoscopy. BMC Pulm Med 2016;16:60.

7. Appelbaum L, Sosna J, Nissenbaum Y, et al. Electromagnetic navigation system for CT-guided biopsy of small lesions. AJR Am J Roentgenol 2011;196:1194-200.
8. Attivissimo F, Lanzolla AML, Carlone S, et al. A novel electromagnetic tracking system for surgery navigation. Comput Assist Surg (Abingdon) 2018;23:42-52.

9. Mehta AC, Hood KL, Schwarz Y, et al. The Evolutional History of Electromagnetic Navigation Bronchoscopy: State of the Art. Chest 2018;154:935-47.

10. Gupta S, Wallace MJ, Cardella JF, et al. Quality improvement guidelines for percutaneous needle biopsy. J Vasc Interv Radiol 2010;21:969-75.

11. Kauczor HU, Baird AM, Blum TG, et al. ESR/ERS statement paper on lung cancer screening. Eur Radiol 2020;30:3277-94.

12. Nakatani M, Tanigawa N, Kariya S, et al. Analysis of factors influencing accuracy and complications in CTguided lung biopsy. Minim Invasive Ther Allied Technol 2012;21:415-22.

13. Tuna T, Ozkaya S, Dirican A, et al. Diagnostic efficacy of computed tomography-guided transthoracic needle aspiration and biopsy in patients with pulmonary disease. Onco Targets Ther 2013;6:1553-7.

14. Andersen FD, Degn KB, Riis Rasmussen T. Electromagnetic navigation bronchoscopy for lung nodule evaluation. Patient selection, diagnostic variables and safety. Clin Respir J 2020;14:557-63.

15. Grasso RF, Faiella E, Luppi G, et al. Percutaneous lung biopsy: comparison between an augmented reality CT navigation system and standard CT-guided technique. Int J Comput Assist Radiol Surg 2013;8:837-48.

16. Wood BJ, Locklin JK, Viswanathan A, et al. Technologies for guidance of radiofrequency ablation in the multimodality interventional suite of the future. J Vasc Interv Radiol 2007;18:9-24.

17. Yarmus LB, Arias S, Feller-Kopman D, et al. Electromagnetic navigation transthoracic needle aspiration for the diagnosis of pulmonary nodules: a safety and feasibility pilot study. J Thorac Dis 2016;8:186-94.

18. Santos RS, Gupta A, Ebright MI, et al. Electromagnetic navigation to aid radiofrequency ablation and biopsy of lung tumors. Ann Thorac Surg 2010;89:265-8.

19. Grand DJ, Atalay MA, Cronan JJ, et al. CT-guided percutaneous lung biopsy: comparison of conventional CT fluoroscopy to CT fluoroscopy with electromagnetic navigation system in 60 consecutive patients. Eur J Radiol 2011;79:e133-6.

20. Wiener RS, Schwartz LM, Woloshin S, et al. Populationbased risk for complications after transthoracic needle lung biopsy of a pulmonary nodule: an analysis of discharge records. Ann Intern Med 2011;155:137-44. 
21. Hiraki T, Mimura H, Gobara H, et al. Incidence of and risk factors for pneumothorax and chest tube placement after CT fluoroscopy-guided percutaneous lung biopsy: retrospective analysis of the procedures conducted over a 9-year period. AJR Am J Roentgenol 2010;194:809-14.

Cite this article as: Zhang $\mathrm{Y}$, Huang $\mathrm{H}$, Cao S, Teng J, Wang Y, Ling X, Zhou Y, Xu J, Li W, Zhong H. Clinical value of an electromagnetic navigation system for CT-guided percutaneous lung biopsy of peripheral lung lesions. J Thorac Dis 2021;13(8):4885-4893. doi: 10.21037/jtd-21-395 